\title{
Endocrine Surgery: Diagnostic Tools with Doubtful Findings, and Rare Tumors of the Adrenal Gland
}

\author{
Koutelidakis I
}

Endocrine surgery is an important part of general surgery as it includes organs that are vital for the function of the human body. In the last ten years research has been focused on the correct diagnosis and recognition of endocrine diseases that were not so extensively examined in the past. We can now be more positively selective when we decide to operate on a thyroid gland, and we can afford to be aggressive when we see a growing tumor of the adrenal gland, because we now understand that growing tumors may be benign, although causing pain and discomfort because of rapid growth.

In the article of Gunes M.E., entitled: Comparision of Preoperative and Postoperative Histopathology Findings in Patients Undergoing Thyroidectomy, who made a postoperative evaluation of the preoperative fine needle aspiration (FNA) thyroid biopsy results after thyroidectomy, a rather high negative predictive value of the FNA was reported [1]. The incidence of non-diagnostic biopsies was also rather high, although in the literature it is reported at approximately 5\%. The current strategy for non-diagnostic FNA results for nodules that are suspicious on ultrasound (US), is not to operate immediately, but to repeat the FNA within a month. Another interesting finding of the above study was the low specificity of FNA, although it has been reported above $85 \%$ in multiple recent studies [2].

Along with thyroid US, FNA is a diagnostic tool much used in the diagnosis of thyroid cancer. The main problem with the cytological results is the finding of cells with atypia, which is an indeterminate result. The current guidelines suggest a repeat biopsy rather than immediate operation, because the final diagnosis of cancer after thyroidectomy, in such cases, is approximately $15 \%$. In conclusion we suggest careful evaluation of the results of the FNA and not to be aggressive, but to delay operation until the picture is clearer, because if the first operation is the right one the prognosis is very good $[3,4]$.

An interesting case report in this issue is the article of Mondal A, et al, entitled: Bilateral Adrenal Myelolipoma:

\footnotetext{
Koutelidakis I

Associate Professor of Surgery, $2^{\text {nd }}$ Department of Surgery School of Medicine, Aristotle University of Thessaloniki

Corresponding author: Koutelidakis Ioannis e-mail: iokoutel@gmail.com
}

A Case Report. They present the case of masses located in both adrenal glands. The rarity of this case is that the final diagnosis was bilateral myelolipoma [5]. The right sided tumor was large enough tocause the mild symptoms that led to the diagnosis and surgery. Adrenal tumors are rare.. and they may be functioning or non-functioning. The non-functioning tumors are mainly "incidentalomas" [6].

Adrenal incidentalomas are masses in the adrenals that are found incidentally on a computed tomography (CT) scan. Their incidence is $4 \%$, their size is usually under $4 \mathrm{~cm}$, and in the vast majority of cases they are nonfunctioning, but rarely subclinical pheochromocytoma or subclinical Cushing's tumor is sometimes diagnosed. In order to decide whether a symptom-free tumor should be removed, evaluation must be made of its functionality. In addition, it must be monitored to observe how much the mass grows in a year. If the mass is large and causes symptoms should be operated on; the size that usually causes symptoms due to pressure of the adjacent organs is $8-10 \mathrm{~cm} \mathrm{[6].}$

The gold standard method of operation for these tumors is laparoscopic, and the open method should be used only for large masses. The cut-off size for excluding the laparoscopic approach is $8 \mathrm{~cm}$, although some reports suggest that laparoscopic extraction of a $10 \mathrm{~cm}$ tumor is feasible. The laparoscopic approach may be either through the peritoneal cavity or retroperitoneal. The open approach may also be through the peritoneal cavity or the retroperitoneal, but for bilateral tumors or unilateral tumors over $10 \mathrm{~cm}$ in size, the intraperitoneal approach is recommended.

Myelolipomas are very rare, comprising approximately $0.8-1 \%$ of adrenal tumors. They are benign tumors and they are usually diagnosed when they grow over $10 \mathrm{~cm}$ and cause pressure symptoms. The treatment is surgical and if they are under $8 \mathrm{~cm}$ in size they should be extracted laparoscopically, otherwise through an open incision [8].

The first article about FNA biopsy reflects the problems that arise if the decision about operating or not on a thyroid gland is taken without second thoughts. The message is that the diagnostic tools in the assessment of thyroid masses must be evaluated appropriately, and if there is any doubt the diagnostic tests should be repeated. In the second article, the main point is that adrenal masses over $10 \mathrm{~cm}$ in size should be removed by the open method, and through the peritoneal cavity. 


\section{Reference}

1. Gunes ME. Comparision of Preoperative and Postoperative Histopathology Findings in Patients Undergoing Thyroidectomy. Hellenic J Surg 2017;89:153-9.

2. Suh CH, Baek JH, Lee JH, et al. The role of core-needle biopsy in the diagnosis of thyroid malignancy in 4580 patients with 4746 thyroid nodules: A systematic review and meta-analysis. Endocrine. 2016;54:315-28.

3. Remonti LR, Kramer CK, Leitão CB, et al. Thyroid ultrasound features and risk of carcinoma: A systematic review and metaanalysis of observational studies. Thyroid. 2015;25:538-50.

4. Baloch ZW, LiVolsi VA. Current role and value of fine-needle aspiration in nodular goitre. Best Pract Res Clin Endocrinol Metab. 2014;28:531-44.

5. Mondal A, Chakrabarti DK, Basu A, et al. Bilateral Adrenal Myelolipoma: A Case Report. Hellenic J Surg 2017;89:160-2.

6. Lam AK. Update on adrenal tumours in 2017 World Health Organization (WHO) of endocrine tumours. Endocr Pathol 2017. doi: 10.1007/s12022-017-9484-5.

7. Maccora D, Walls GV, Sadler GP, et al. Bilateral adrenalectomy: A review of 10 years' experience.Ann R Coll Surg Engl 2017;99:119-22.

8. Davide Zattoni, Ruben Balzarotti, Raffaele Rosso. The management of bilateral myelolipoma: Case report and review of the literature. Int J Surg Case Rep 2015;12:31-6. 